\title{
Dispersion relation for electron waves propagating in an isotropic plasma containing Maxwellian and suprathermal electrons
}

\author{
By JUAN R. SANMARTIN \\ Instituto Nacional de Téenica Aeroespacial 'Esstcban Terradas', \\ Madrid, Spain
}

(Received 10 June 1974 and in revised form 8 October 1974)

The paper discusses the dispersion relation for longitudinal electron waves propagating in a collisionless, homogeneous isotropic plasma, which contains both Maxwellian and suprathermal electrons. It is found that the dispersion curve, known to have two separate branches for zero suprathermal energy spread, depends sensitively on this quantity. As the energy half-width of the suprathermal population increases, the branches approach each other until they touch at a connexion point, for a small critical value of that half-width. The topology of the dispersion curves is different for half-widths above and bolow critical; and this can affect the use of wave-propagation measurements as a diagnostic technique for the determination of the electron distribution function. Both the distance between the branches and spatial damping near the connexion frequency depend on the half-width, if below eritical, and can be used to determine it. The theory is applied to experimental data.

\section{Introduction}

Henry, Treguier and co-workers (Le Coquil et al. 1971; Treguier \& Henry 1972; Henry \& Treguier 1972a, $b, 1973$ ) discussed the propagation of longitudinal, clectron waves in a plasma, as a way to obtain information about its electron distribution function $f(v)$. They used a hot, unmagnetized diffusion plasma under collisionless, quasi-homogeneous conditions to study experimentally the appropriate dispersion relation $D(\mathbf{k}, \omega)=0$ for real frequency $\omega$ and complex wavenumber $\mathbf{k}$.

The theoretical curves $\operatorname{Re} k(\omega), \operatorname{Im} k(\omega)$ for $f(\mathrm{v})$ Maxwellian are well known (Landau 1946; Kuehl, Stewart \& Yeh 1965). Comparison with the corresponding experimental curves may serve as a diagnostic technique for the electron density and temperature $n_{e}$ and $T_{e}$. The Maxwellian $R e k(\omega)$ (shown schematically in figure 1: dashed line $A D) \dagger$ exists only for $\omega>\omega_{p}$, where $\omega_{p}$ is the plasma frequency. In the experiments of Henry \& Treguier, nonetheless, propagation for $\omega<\omega_{p}$ was detected under particular conditions.

It was initially assumed that the unexpected frequencies corresponded to

$\uparrow$ The dispersion relation for a Maxwellian plasma has an infinity of roots. We shall always speak, as is usual, of the least-damped root. 


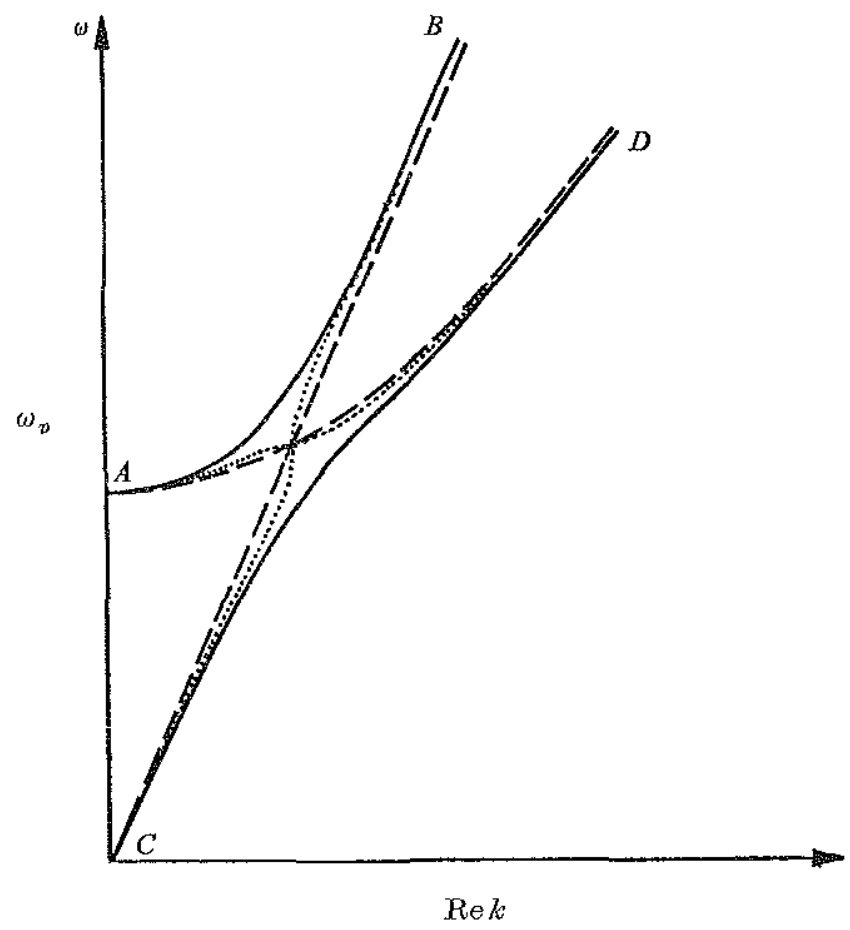

FrguRE 1. Dispersion curves for electron waves: Maxwellian population of electrons, ---$(A D)$; Maxwellian plus suprathermal populations, - - (zero suprathermal half-width), ...... (half-width above critical).

electron ballistic waves (Treguier \& Henry 1972; Henry \& Treguier 1972a). Independent measurements of $f(v)$ later cleared up the phenomenon. It was shown that $f(v)$ differed from a Maxwellian distribution, in that there existed a small isotropic population of electrons with the same (suprathermal) energy of the ionizing electrons in the discharge producing the plasma (Henry \& Treguier $1972 b, 1973)$. Propagation below $\omega_{p}$ was detected only when the thermal and suprathermal populations were well differentiated. Assuming that

$$
f(\mathbf{v})=f_{1}+f_{2}
$$

where $f_{1}$ and $f_{2}$ are Maxwellian and suprathermal (mono-energetic) distributions, respectively, and choosing appropriately the density and energy of $f_{2}$, Henry \& Treguier solved the dispersion relation, and obtained good agreement with the experimental results. Figure 1 shows schematically $\operatorname{Re} k(\omega)$ for such a distribution function (solid lines). There are two branches. Branch $A B$ approaches the usual Maxwellian curve for small Re $k$, and the straight line $\omega /(\operatorname{Re} k)=v_{c}$ (the velocity corresponding to the suprathermal energy) for large Re $k$. Branch $C D$ approaches $\omega /(\operatorname{Re} k)=v_{c}$ for small Re $k$, and the Maxwellian curve for large Re $k$.

The analysis and results of Henry \& Treguier are of importance for many experimental situations. $\uparrow$ Nonetheless, these anthors left aside an interesting:

I A main oxample is the ionospheric plasma, where there exists a suprathormal population of photoelectrons. 
aspect of the problem: they always assumed $f_{2}$ to be mono-energetic; and they simply noticed, in this respect, that a non-vanishing energy spread in $f_{2}$ would affect $\operatorname{Im} k(\omega)$ along the branch $A B$. In the present paper, on the other hand, it is shown that, even if $f_{2}$ is a narrow distribution, to consider it mono-energetic may be inadequate, because $k(\omega)$ is a function sensitive to small variations in the spread of that distribution.

The half-width of $f_{2}(v)$ is a measure of that spread. As this half-width is increased, starting from zero, branches $A B$ and $C D$ are found to approach cach other. The difference between the values of $\operatorname{Re} k(\omega)$ on the two branches has a minimum at a certain frequency $\omega_{0}$, and although the minimum depends on the half-width, $\omega_{0}$ does not; furthermore, both branches have a half-width dependent, common value of $\operatorname{Im} k\left(\omega_{0}\right)$. As the half-width is further increased, a critical value is reached for which that minimum vanishes; the two branches then touch each other, since they have common values of $\operatorname{Re} k\left(\omega_{0}\right)$ and of $\operatorname{Im} k\left(\omega_{0}\right)$. For halfwidths above critical the branches again separate; they still hold a common value of $\operatorname{Re} k\left(\omega_{0}\right)$, but take differing values of $\operatorname{Im} k\left(\omega_{0}\right)$. Thus, the topology of the dispersion relation in the three-dimensional space $(\omega, \operatorname{Re} k, \operatorname{Im} k)$ experiences a non-trivial change in the transition through the critical half-width. Above it, and as seen in the plane $(\omega, \operatorname{Re} k)$, point $A$ is connected to $D$, and $C$ is connected to $B$ (figure 1, dotted lines). Somewhat similar results are found in a problem studied by O'Neil \& Malmberg (1968), in which a quiescent thermal plasma coexists with a low-density, high-velocity electron beam.

This dependence of the $\omega, k$ topology on the suprathermal half-width should be of importance in using electron wave propagation as a diagnostic technique. But, apart from this, the half-width may be a parameter of experimental interest in itself. From the preceding paragraph, it appears that it can be obtained from measurements of $\operatorname{Im} k\left(\omega_{0}\right)$ and of $\operatorname{Re} k\left(\omega_{0}\right)$ in both branches.

The present paper deals with this curious behaviour of the dispersion branches. In $\S 2$, choosing a simple form of $\int_{2}(v)$, we discuss the dispersion relation for frequencies not close to $\omega_{0}$. In $\S 3$ we carry out a detailed study of the frequency region near $\omega_{0}$. We discuss, and analytically determine, the evolution of the $\omega, k$ topology with the increase in the half-width, from zero to values above critical. To investigate the dependence of the results upon the detailed form of $f_{2}$ (for a given half-width), we briefly consider in $\$ 4$ two other forms of $f_{2}$. In $\$ 5$ we discuss the results and apply them to the available experimental data.

\section{The dispersion relation for a Lorentzian type of suprathermal distribution}

Let the electron distribution function be $f=f_{1}+f_{2}$, where

$$
f_{1}=n_{1} \pi^{-\frac{3}{2}} u^{-3} \exp \left(-v^{2} / u^{2}\right), \quad f_{2}=n_{2} \phi(v),
$$

$u=\left(2 k_{B} T_{e} / m\right)^{\frac{1}{2}}, \phi$ is isotropic and normalized to unity, and all symbols have their usual meaning. The dispersion relation for longitudinal electron waves then reads

$$
1-\frac{\omega_{p 1}^{2} Z^{\prime}(\omega / k u)}{k^{2}}-\frac{\omega_{p 2}^{2}}{k^{2}} \int_{c} \frac{d v_{z} d \bar{\phi} / d v_{z}}{v_{z}-\omega / k}=0 .
$$


Here $Z$ is the Fried \& Conte (1961) function, $\omega$ (real) and $\mathbf{k}$ (complex) are the frequency and wavenumber of the waves, and the integral follows the usual Landau contour. We have defined

$$
\omega_{p j}^{2} \equiv 4 \pi n_{j} e^{2} / m \text { and } \bar{\phi}\left(v_{z}\right) \equiv \int \phi(v) d v_{x} d v_{y}
$$

(the $z$ axis being taken along $\mathbf{k}$ ).

Henry \& Treguier $(1972 b, 1973)$ assumed $\phi$ to be mono-energetic,

$$
\phi=\left(4 \pi v_{c}^{2}\right)^{-1} \delta\left(v-v_{c}\right),
$$

so that $\bar{\phi}$ is a 'water-bag' distribution

$$
\bar{\phi}=\left(2 v_{c}\right)^{-i}\left[H\left(v_{z}+v_{c}\right)-H\left(v_{z}-v_{c}\right)\right],
$$

$H$ being the Heaviside function. In that case $f_{2}$ is characterized by just two parameters, $n_{2}$ and $v_{c}$, or non-dimensionally by

$$
\alpha=n_{2} / n_{1} \approx n_{2} / n_{e}, \quad \theta=v_{e}^{2} / u^{2},
$$

where $\alpha$ and $\theta^{-1}$ are assumed to be small. On the other hand, if $\phi$ is a narrow-peak distribution (but not a $\delta$ function) centred at $v_{c}$, there exists a third parameter of interest, the half-width (full width at half-height) of the peak $2 \Delta v_{2} \uparrow \uparrow$ Then, defining

$$
\sigma \equiv \frac{\Delta v_{2}}{v_{c}}, \quad A\left(\frac{\omega}{k u}, \theta, \sigma\right) \equiv \frac{k^{2}}{\omega^{2}}\left(\int_{c} \frac{d v_{z} d \bar{\phi} / d v_{z}}{v_{z}-\omega / k}\right)^{-1},
$$

we may rewrite (2) in the non-dimensional form

$$
\left[\left(\frac{\omega}{\omega_{p 1}}\right)^{2}-\left(\frac{\omega}{k u}\right)^{2} Z^{\prime}\left(\frac{\omega}{k u}\right)\right] A\left(\frac{\omega}{k u}, \theta, \sigma\right)=\alpha .
$$

In the mono-energetic limit $(\sigma \rightarrow 0)$, we have $A \rightarrow 1-\theta(k u / \omega)^{2}$.

We shall take $\phi$ in as simple a form as possible. + Now, notice that, calling $v_{\perp}=\left(v_{x}^{2}+v_{y}^{2}\right)^{\frac{1}{2}}$, we have

$$
\bar{\phi}\left(v_{z}\right)=2 \pi \int_{0}^{\infty} v_{\perp} d v_{\perp} \phi(v)=2 \pi \int_{\left\lfloor v_{z} \mid\right.}^{\infty} v d v \phi(v),
$$

and therefore

$$
d \bar{\phi} / d v_{s}=-2 \pi v_{s} \phi\left(\left|v_{s}\right|\right) \text {. }
$$

Just for convenience, we choose $\phi$ to be formally an even function of $v$; in this way,

$$
d \bar{\phi} / d v_{z}=-2 \pi v_{z} \phi\left(v_{z}\right)
$$

This simplifies the analytical evaluation of $A$. Actually, it is clear that, given any distribution $\phi(v)$ that nearly vanishes outside a narrow interval around $v_{c}$, one can get an even distribution which nearly vanishes outside that interval, and is practically identical to $\phi(v)$ inside it, viz. $\phi(v)+\phi(-v)$. We now choose the distribution

$$
\phi^{\mathrm{I}}=\frac{v_{B}}{4 \pi^{2} v_{c} v}\left[\frac{1}{v_{B}^{2}+\left(v-v_{c}\right)^{2}}-\frac{1}{v_{B}^{2}+\left(v+v_{c}\right)^{2}}\right],
$$

$\dagger$ The energy half-width is obviously $\left(2 \Delta v_{2}\right) m v_{c}$.

+ For $f_{2}$ narrow, it appears reasonable to assume that the dispersion relation will depend mainly on $\alpha, \theta$ and $\sigma$, and only weakly on the cletailed shape of the peak. We come back to this point later. 
for which $\Delta v_{2} \approx v_{B}$. It is easy to find the results

$$
\left.\begin{array}{l}
\bar{\phi}^{\mathrm{I}}=\frac{1}{2 \pi v_{c}}\left[\ln ^{-1}\left(\frac{v_{z}+v_{c}}{v_{B}}\right)-t n^{-1}\left(\frac{v_{z}-v_{c}}{v_{B}}\right)\right], \\
A^{\mathrm{X}}=\left(1+i \sigma 0 \frac{1}{2} \frac{k u}{\omega}\right)^{2}-0\left(\frac{k u}{\omega}\right)^{2} .
\end{array}\right\}
$$

Equation (7) is to be solved assuming $\alpha, 0^{-1}$ and $\sigma$ small. For small $\alpha$ there are two distinct roots, or branches, which can be determined immediately in an approximate way. First, we write

$$
\left(\frac{\omega}{\omega_{p 1}}\right)^{2}-\left(\frac{\omega}{k u}\right)^{2} Z^{\prime}\left(\frac{\omega}{k u}\right)=\frac{\alpha}{A^{\mathrm{I}}(\omega / k u, 0, \sigma)} .
$$

For $\omega / k u$ real and large, $\operatorname{Im} Z^{\prime}(\omega / k u)$ is very small. We neglect both $\alpha$ and $\operatorname{Im} Z^{\prime}(\omega / k u)$, and get a real root (i.e. $\operatorname{Im} k=0$ ),

$$
\frac{\omega}{\omega_{p 1}} \approx \frac{\omega}{k u}\left[\operatorname{Re} Z^{\prime}\left(\frac{\omega}{k u}\right)\right]^{\frac{1}{2}},
$$

the usual Landau root. Second, we write

$$
A^{\mathrm{I}}\left(\frac{\omega}{k u}, \theta, \sigma\right)=\frac{\alpha}{\left(\omega / \omega_{p 1}\right)^{2}-(\omega / k u) \overline{Z^{\prime}(\omega / k u)}} ;
$$

and, neglecting both $\alpha$ and $\sigma$, we get a real root again,

$$
\omega \approx v_{c} k \text {. }
$$

Equations (13) and (15) correspond to the dashed lines of figure 1. When $\theta^{-1}$ is small, these equations have a common root $\left(\omega_{0}, k_{0}\right)$

$$
\omega_{0}=\omega_{p 1}\left[\theta \operatorname{Re} Z^{\prime}\left(0^{\frac{1}{2}}\right)\right]^{\frac{1}{2}}, \quad k_{0}=\omega_{0} / v_{c} .
$$

Near this point, the right-hand sides of (12) and (14) cannot be neglected; and the approximations (13) and (15) cease to be valid. These approximations describe well parts $A, D$ and $B, C$, respectively, of the solid and dotted lines of figure 1 .

\section{3. $\omega, k$ topology near $\omega_{0}$}

Detailed analysis is required for the neighbourhood of the point defined in (16). We write

$$
\omega=\omega_{0}+\delta \omega, \quad k=k_{0}+\delta k ;
$$

and we linearize (7) around $\omega_{0}, k_{0}$. Notice, from (16), that

thus

$$
\begin{gathered}
\frac{\omega}{k u} \approx \theta^{\frac{1}{2}}+\theta^{\frac{x}{2}}\left(\frac{\delta \omega}{\omega_{0}}-\frac{\delta k}{k_{0}}\right) ; \\
A^{\mathrm{I}} \approx\left[1+i \sigma\left(1-\frac{\delta \omega}{\omega_{0}}+\frac{\delta k}{k_{0}}\right)\right]^{2}-\left(1-2 \frac{\delta \omega}{\omega_{0}}+2 \frac{\delta k}{k_{0}}\right) \\
\approx 2\left(\frac{\delta \omega}{\omega_{0}}-\frac{\delta k}{k_{0}}+i \sigma\right),
\end{gathered}
$$


since $\sigma \ll 1$. Similarly we have

So

$$
\begin{aligned}
Z^{\prime}\left(\frac{\omega}{\kappa u}\right) & \approx Z^{\prime}\left(\theta^{\frac{1}{2}}\right)+\left(\frac{\delta \omega}{\omega_{0}}-\frac{\delta k}{k_{0}}\right) \theta^{\frac{1}{2}} Z^{\prime \prime}\left(\theta^{\frac{1}{2}}\right) \\
& =Z^{\prime}\left(\theta^{\frac{1}{2}}\right)+\left(\frac{\delta \omega}{\omega_{0}}-\frac{\delta k}{k_{0}}\right)\left[2+(1-2 \theta) Z^{\prime}\left(\theta^{\frac{1}{2}}\right)\right] .
\end{aligned}
$$

$$
\left(\frac{\omega}{\omega_{p 1}}\right)^{2}-\left(\frac{\omega}{k u}\right)^{2} Z^{\prime}\left(\frac{\omega}{k u}\right) \approx P(\theta) \frac{\delta \omega}{\omega_{0}}-Q(\theta) \frac{\delta k}{k_{0}}-i \theta \operatorname{Im} Z^{\prime}\left(\theta^{\frac{1}{2}}\right)
$$

where we define

$$
\begin{aligned}
& P(\theta) \equiv(2 \theta-1) \theta \operatorname{Re} Z^{\prime}\left(\theta^{\frac{1}{2}}\right)-2 \theta, \\
& Q(\theta) \equiv P(\theta)-2 \theta \operatorname{Re} Z^{\prime}\left(\theta^{\frac{1}{2}}\right),
\end{aligned}
$$

and neglect products of $\operatorname{Im} Z^{\prime}\left(\theta^{\frac{1}{2}}\right)$, which represents the usual Landau damping, with either $\delta \omega / \omega_{0}$ or $\delta k / k_{0} \cdot \dagger$

Introducing (18) and (19) in (7), we get a quadratic equation for $\delta k$. We write it in non-dimensional form by defining appropriate scaled wavenumber and frequency

$$
y^{\mathrm{T}}=[2 Q(\theta) / \alpha]^{\frac{1}{2}} \delta k / k_{0}, \quad x=P(\theta)[2 / \alpha Q(\theta)]^{\frac{1}{2}} \delta \omega / \omega_{0},
$$

and scaled half-width and Landau damping

We get

$$
\nu=[2 Q(\theta) / \alpha]^{\frac{1}{2}} \sigma, \quad \mu=[8 \pi / \alpha Q(\theta)]^{\frac{1}{2}} \theta^{\frac{3}{2}} \exp (-\theta) .
$$

$$
\left(y^{\mathrm{I}}-x-i \mu\right)\left(y^{\mathrm{I}}-x Q / P-i \nu\right)=1 .
$$

This equation yields $\delta k / k_{0}$ as a function of $\delta \omega / \omega_{0}, \alpha, \theta$ and $\sigma$, and describes the topology of the dispersion relation near $\omega_{0}, k_{0}$. We shall assume $Q / P<1$ and $\mu<2$; actually $Q / P$ is small for large $\theta$, and $\mu$ is, too, unless $\alpha$ is extremely small.

Equation (25) has two roots, $y_{1}^{\mathrm{I}}(x)$ and $y_{2}^{\mathrm{I}}(x)$. As $x \rightarrow-\infty$, they approach, for all $\nu$, either $(x+i \mu)$ or $(x Q / P+i \nu)$. We shall call $y_{1}^{\mathrm{T}}$ the root that approaches $(x+i \mu)$. It is then easy to show that

$$
y_{2}^{\mathrm{T}}-y_{1}^{\mathrm{I}}=\left[4-(\nu-\mu)^{2}+x^{2}(1-Q / P)^{2}-2 i x(y-\mu)(1-Q / P)\right]^{\frac{1}{2}} .
$$

Thus both roots coalesce for

$$
x=0, \quad \nu=\nu_{*}^{\mathrm{I}}(\mu) \equiv 2+\mu .
$$

It may also be seen from (26) that, for $\nu<\nu_{*}^{\mathrm{I}}, \operatorname{Re} y_{2}^{\mathrm{I}}-\operatorname{Re} y_{1}^{\mathrm{I}}$ has a minimum, as a function of $x$, at $x=0$. Call it $\Delta\left(\operatorname{Re} y^{\mathrm{I}}\right)$; then

$$
\Delta\left(\operatorname{Re} y^{\mathrm{I}}\right)=\left[4-(\nu-\mu)^{2}\right]^{\frac{t}{2}} .
$$

For $x=0$, the roots are very simple:

$$
\begin{aligned}
& y_{1}^{\Upsilon}(x=0)=i \frac{1}{2}(\nu+\mu)-\frac{1}{2}\left[4-(\nu-\mu)^{2}\right]^{\frac{1}{2}}, \\
& y_{2}^{\Upsilon}(x=0)=i \frac{1}{2}(\nu+\mu)+\frac{1}{2}\left[4-(\nu-\mu)^{2}\right]^{\frac{1}{2}} .
\end{aligned}
$$

$\dagger$ From the well.known behaviour of $Z$ we have $P \approx 2, Q \approx 3 / \theta$ for very large $\theta$, and $\operatorname{Im} Z^{\prime}\left(\theta^{\frac{1}{2}}\right)=-2 \pi^{\frac{1}{2}} \theta^{\frac{1}{2}} \exp (-\theta)$, which is very small for large $\theta$. 


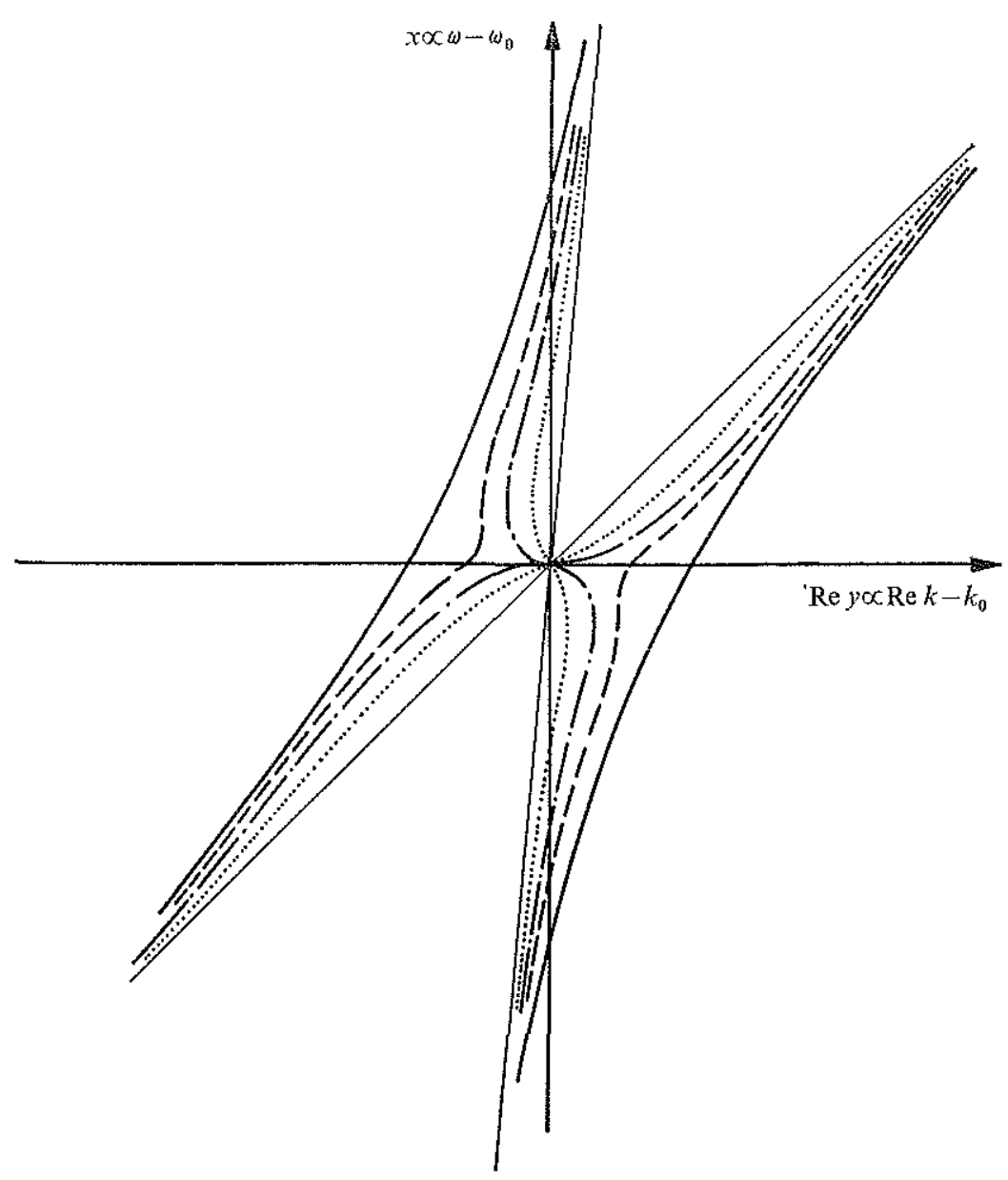

FrGURE 2. Dispersion curves near the connexion point $\omega_{0}, k_{0} . \nu$ is the normalized suprathermal half-width, $\nu^{*}$ its critical value: $\nu=0, \longrightarrow ; 0<\nu<\nu^{*},--; \nu=\nu^{*}, \ldots . \ldots ; \nu>\nu^{*}, \ldots$

Therefore, at $x=0$ and for $\nu<\nu_{*}^{\mathrm{I}}$, the two roots have a common imaginary part. Call it $\operatorname{Im}\left(y^{\mathrm{I}}\right)$; then

$$
\operatorname{Im}\left(y^{\mathrm{I}}\right)=\frac{1}{2}(\nu+\mu)
$$

which increases with $\nu$. For $\nu>\nu_{*}^{\mathrm{I}}$, both $y_{1}^{\mathrm{I}}(x=0)$ and $y_{2}^{\mathrm{I}}(x=0)$ become purely imaginary; then $\Delta\left(\operatorname{Re} y^{I}\right)=0$ (at $\left.x=0\right)$, while $\operatorname{Im} y_{1}^{\mathrm{I}}(x=0) \neq \operatorname{Im} y_{2}^{\mathrm{I}}(x=0)$.

We are now in a position to discuss the topology of the dispersion curves. For $\nu=0$, one should recover the mono-energetic limit of Henry \& Treguier. Thus $\operatorname{Re} y_{1}^{\mathrm{T}}$ and $R e y_{2}^{\mathrm{T}}$ must behave as shown schematically in figure 2 (solid lines). One can show that this is indeed the case: $y_{1}^{\mathrm{I}} \rightarrow x Q / P+i \nu$ as $x \rightarrow+\infty$, while $y_{2}^{\mathrm{I}}$ approaches $x Q / P+i \nu$ as $x \rightarrow-\infty$, and $x+i \mu$ as $x \rightarrow+\infty$. For $\nu=\nu$, the branches have common values of both $\operatorname{Re} y^{\mathrm{I}}$ and $\operatorname{Im} y^{\mathrm{I}}$. That is, they touch each other in the three-dimensional space $(x, \operatorname{Re} y, \operatorname{Im} y)$. In the plane $(x, \operatorname{Re} y)$ they behave as shown in figure 2 (dashed-dotted lines). For $\nu$ increasing from zero to $\nu_{*}^{\mathrm{I}}$, the branches approach each other; but they show the same topology of the $\nu=0$ 


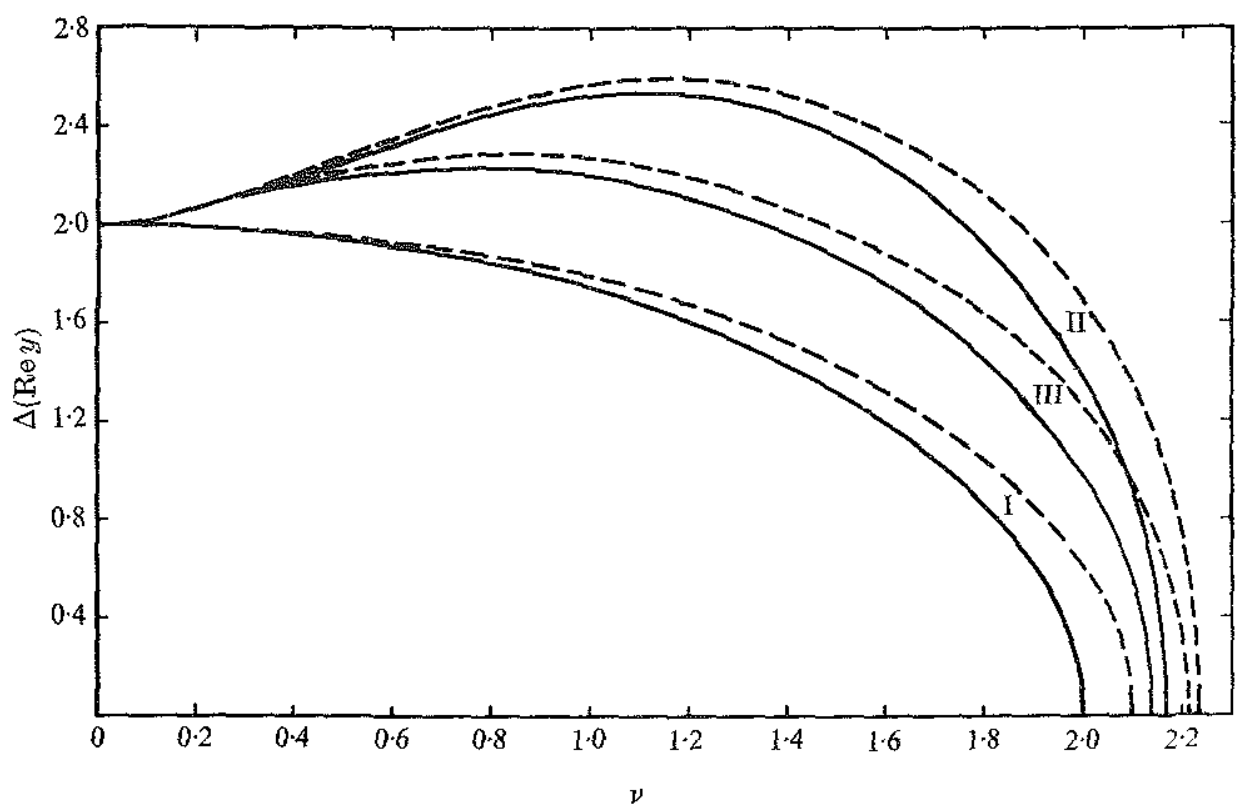

Frgurg 3. Normalized difference between the wavenumbers at $\omega_{0}$ for the two branches $\Delta\langle$ Re $y\rangle$ against $\nu$ and $\mu$ (a measure of usual Landau dampingnear $\left.\omega_{0}, k_{0}\right\rangle . \mu=0, \ldots ; \mu=0 \cdot 1$, -- - For suprathermal distributions I, II and III.

limit. They behave as shown by the dashed lines of figure 2. For $\nu>\nu_{*}^{\mathrm{I}}$, the branches again separate; but now they have equal values of Re $y^{\mathrm{I}}$ and differing values of $\operatorname{Im} y^{I}$. Thus they cross each other in the plane $(x, \operatorname{Re} y)$ (figure 2, dotted lines). As $x \rightarrow+\infty$, we have $y_{1}^{x} \rightarrow x+i \mu, y_{2}^{\frac{T}{2}} \rightarrow x Q / P+i \nu$. There is, in conclusion, an important topological change at the transition through the critical value $\nu=\nu_{*}^{\mathrm{I}}$.

\section{Other suprathermal distributions}

To find out how general the preceding results are, we briefly consider in this section two other forms of $\phi$ :

$$
\begin{gathered}
\phi^{\mathrm{II}}=\frac{1}{4 \pi^{\frac{3}{2} v_{B} v_{c} v}}\left[\exp \left\langle-\frac{-\left(v-v_{c}\right)^{2}}{v_{B}^{2}}\right\rangle-\exp \left\langle\frac{-\left(v+v_{c}\right)^{2}}{v_{B}^{2}}\right\rangle\right], \\
\phi^{\mathrm{III}}=\frac{v_{B}^{3}}{2 \pi^{2} v_{c} v}\left[\frac{1}{\left\langle v_{B}^{2}+\left(v-v_{c}\right)^{2}\right\rangle^{2}}-\frac{1}{\left\langle v_{B}^{2}+\left(v+v_{c}\right)^{2}\right\rangle^{2}}\right],
\end{gathered}
$$

for which $\Delta v_{2} \approx(\ln 2)^{\frac{1}{2}} v_{B} \approx 0 \cdot 83 v_{B}$ and $\Delta v_{2} \approx\left(2^{\frac{1}{2}}-1\right)^{\frac{1}{2}} v_{B} \approx 0 \cdot 64 v_{B}$, respectively. It is easy to determine that (13) and (15) are valid for $\phi^{\mathrm{II}}$ and $\phi^{\mathrm{III}}$ as well as for $\phi^{\mathrm{I}}$. We again find that, for the neighbourhood of the point defined in (16), a detailed analysis is needed. Proceeding as in $\S 3$, we arrive at

$$
\begin{gathered}
y^{\mathrm{II}}-x-i \mu=(0 \cdot 83 / v) Z\left(0.83\left(x Q / P-y^{\mathrm{II}}\right) / \nu\right), \\
\left(y^{\mathrm{III}}-x-i \mu\right)\left(y^{\mathrm{II}}-x Q / P-i \nu / 0 \cdot 64\right)^{2}=y^{\mathrm{II}}-x Q / P-i \nu / 0 \cdot 32
\end{gathered}
$$

for $\phi^{I I}$ and $\phi^{I I I}$, respectively. 


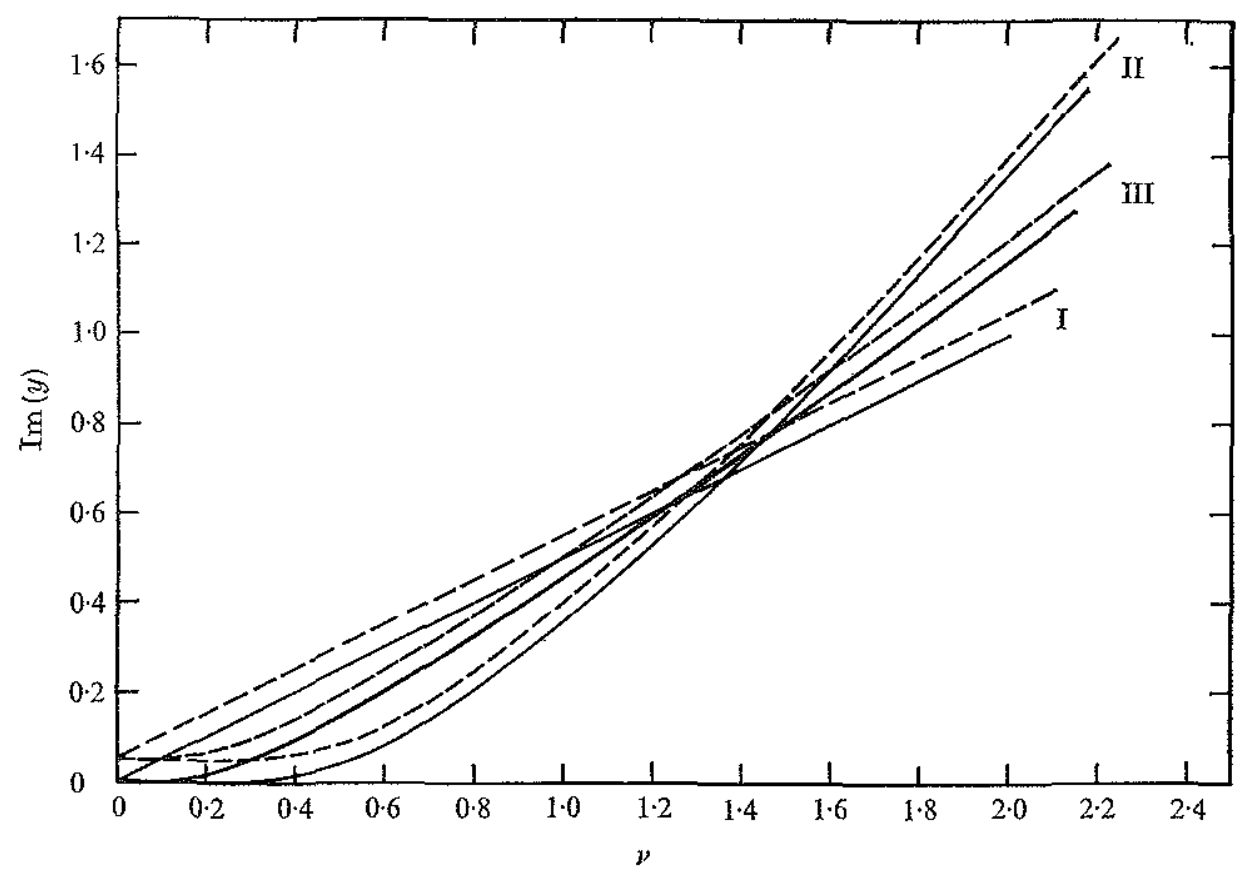

FIGURE 4. Normalized spatial damping, samo for both branches, at $\omega_{0}, \operatorname{Im}(y)$, against $\nu$ and $\mu \cdot \mu=0,-; \mu=0 \cdot 1,---$. For suprathermal distributions I, II and III.

Equation (33) has two roots, $y_{1}^{\mathrm{II}}(x)$ and $y_{2}^{\mathrm{II}}(x)$, which at $x=0$ and at a critical value of $\nu\left(\nu_{*}^{\mathrm{TT}}\right)$, coalesce. For $\nu=0,(33)$ and (25) become identical. For $0<\nu<\nu_{*}^{I I}, \operatorname{Re} y_{2}^{I I}-\operatorname{Re} y_{1}^{I I}$ has a minimum, $\Delta\left(\operatorname{Re} y^{I I}\right)$, at $x=0$. For $0<\nu<\nu_{*}^{I X}$ and at $x=0$, too, $y_{1}^{\mathrm{II}}$ and $y_{2}^{\mathrm{II}}$ have an equal imaginary part $\operatorname{Im}\left(y^{\mathrm{II}}\right)$, and opposite real parts. Thus,

$$
\begin{aligned}
& y_{1}^{\mathrm{II}}(x=0)=i \operatorname{Im}\left(y^{\mathrm{II}}\right)-\frac{1}{2} \Delta\left(\operatorname{Re} y^{\amalg}\right), \\
& y_{2}^{\mathrm{II}}(x=0)=i \operatorname{Im}\left(y^{\mathrm{II}}\right)+\frac{1}{2} \Delta\left(\operatorname{Re} y^{\mathrm{II}}\right) .
\end{aligned}
$$

As $\nu$ increases to $\nu_{*}^{\mathrm{II}}, \operatorname{Im}\left(y^{\mathrm{II}}\right)$ increases, and $\Delta\left(\operatorname{Re} y^{\mathrm{II}}\right)$ decreases to zero. For $\nu>\nu_{*}^{\mathrm{II}}, y_{1}^{\mathrm{II}}(x=0)$ and $y_{2}^{\mathrm{II}}(x=0)$ become purely imaginary; $\Delta\left(\operatorname{Re} y^{\mathrm{II}}\right)$ vanishes (at $x=0$ ) and $\operatorname{Im} y_{1}^{\mathrm{II}}(x=0) \neq \operatorname{Im} y_{2}^{\mathrm{II}}(x=0)$. The $\omega, k$ topology is therefore the same as that of (25). Similar results are found in (34).

The qualitative behaviour just discussed appears to depend only on $\alpha, \theta^{-1}$ and $\sigma$ being small. Quantitative results must be found numerically. For small $\mu$ we find

$$
\nu_{*}^{\mathrm{II}}(\mu) \approx 2 \cdot 17+0 \cdot 7 \mu, \quad \nu_{*}^{\mathrm{III}}(\mu) \approx 2 \cdot 14+0 \cdot 8 \mu,
$$

which should be compared with $\nu_{*}^{\mathrm{I}}$ in (27). Numerioal results for $\Delta\left(\operatorname{Re} y^{I I}\right)$ and $\Delta\left(\operatorname{Re} y^{\mathrm{III}}\right)$ are presented, together with $\Delta\left(\operatorname{Re} y^{\mathrm{I}}\right)$ from (28), in figure 3. Numerical results for $\operatorname{Im}\left(y^{\mathrm{II}}\right)$ and $\operatorname{Im}\left(y^{\mathrm{III}}\right)$, together with $\operatorname{Im}\left(y^{\mathrm{I}}\right)$ from (30), are presented in figure 4 . All these curves end at the appropriate value of $\nu_{*}$. For $\mu$ small, usually the case of interest, $\Delta(\operatorname{Re} y)$ and $\operatorname{Im}(y)$ are linear in $\mu$. Thus we have drawn curves for two values of $\mu$. Notice that neither $\Delta(\operatorname{Re} y)$ nor $\operatorname{Im}(y)$ depends on $Q / P$. 


\section{Conclusions}

The dispersion relation $k(\omega)$ for longitudinal waves in a plasma containing both Maxwellian and suprathermal populations has been found to depend sonsitively on the suprathermal energy half-width. For a zero value of this quantity, the dispersion curves are known to present two separate branches (Henry \& Treguier 1972b). We find here that, when increasing values of the halfwidth are considered, the distance between branches decreases, until at a small critical value of the half-width they touch at a connexion point $\omega_{0}, k_{0}$.

The topology of the dispersion curves is different for half-widths above and below critical; and this should be taken into account when using wave propagation measurements to get information on the electron distribution function. Furthermore, the sensitiveness of the $k(\omega)$ curves to the suprathermal energy spread can be used to measure that spread in an actual situation. Not only the distance between branches, but also the spatial damping $\operatorname{Im} k$ (the same for both branches) near $\omega_{0}$, depends on the suprathermal half-width, if below eritical.

Normalized values for the difference between wavenumbers on the two branches, $\Delta(\operatorname{Re} y)$, and spatial damping, $\operatorname{Im}(y)$, both at $\omega=\omega_{0}$, are given, in figures 3 and 4, against normalized half-width $\nu$, for three different shapes of suprathermal distribution, all with the same half-width $\nu<\nu^{*}$ ( $\nu^{*}$ critical halfwidth). Both $\Delta(\operatorname{Re} y)$ and $\operatorname{Im}(y)$ are also weakly dependent on a parameter $\mu$, a measure of the usual Landau damping near $\omega_{0}, k_{0} . \nu^{*}$ is given in $(27),(35)$ and (36) for the three suprathermal distributions, I, II, and III.

The results depend on the distribution assumed, but this is mostly a weak dependence. Furthermore, distribution I is less realistic than either II or III. The results for just II and III, letting aside I, are quite close, although these distributions are functionally very different from each other. Disagreements are larger for $\Delta(\operatorname{Re} y)$. Anyway, neither $\operatorname{Im} k$, nor the difference between $\operatorname{Re} k$ on the two branches, can be easily measured accurately. Moreover, figures 3 and 4 , for a given $\mu$, yield a relation between $\Delta(\operatorname{Re} y)$ and $\operatorname{Im}(y)$, independently of the value of $\nu$. This may serve as a useful check.

Henry \& Treguier $(1972 b, 1973)$ give experimental data for application. Henry $\&$ Treguier $(1972 b$, figure 2 ) shows experimental values of Re $k$ and $\operatorname{Im} k$. From the figure, the plasma frequency and the mean suprathermal speed are found to be $\omega_{p 1}=5 \pi \times 10^{8} \mathrm{~s}^{-1}$ and $v_{c}=1 \cdot 3 \pi \times 10^{8} \mathrm{~cm} \mathrm{~s}^{-1}$. Also, $\theta \equiv m v_{c}^{2} / 2 k_{B} T_{e}$ and $\alpha$ (the suprathermal-to-Maxwellian density ratio) have taken to be 9 and 0.025 , respectively. These appear, from the paper, to be appropriate approximate values. Anyway, the determination of $\nu$ from $\operatorname{Im} k$ depends only weakly on $\theta$ and $\alpha$. We then find, from (22),

$\operatorname{Im} y \simeq 1.4 \operatorname{Im} k, \quad \Delta(\operatorname{Re} y) \simeq 1.4\left[\operatorname{Re} k_{2}\left(\omega_{0}\right)-\operatorname{Re} k_{1}\left(\omega_{0}\right)\right] \quad($ branches 1 and 2$)$, and, from the experiments, $\operatorname{Im} k \simeq 0.4 \mathrm{~cm}^{-1}$. Then $\operatorname{Im} y \simeq 0.58$, and, from figure 4, $\nu \simeq 1 \cdot 2$. From $(6)$ and $(24)$ we finally get

$$
\Delta v_{2} \simeq 0.19 v_{c},
$$

the suprathermal velocity distribution decreasing by one-half between $v_{c}$ and 
$v_{c} \pm \Delta v_{2}$. As a check, from $\nu \simeq 1.2$ and figure 3 (using curves for II and III), we obtain $\Delta(\operatorname{Re} y) \simeq 2 \cdot 3$, and therefore

$$
\operatorname{Re} k_{2}\left(\omega_{0}\right)-\operatorname{Re} k_{1}\left(\omega_{0}\right) \simeq 1.6 \mathrm{~cm}^{-1},
$$

in reasonable agreement with the experimental data. We also applied our results to data of Henry \& Treguier (1973).

\section{REFERENCES}

Fried, B. D. \& Conte, S. 1961 The Plasma Dispersion Function. Academic.

Henky, D. \& Treguter, J. P. $1972 a$ Phys. Lett. A 38, 115.

Henry, D. \& Trequen, J. P. $1972 b$ J. Plasma Phys. 8, 311.

Hentr, D. \& Treguier, J. P. 1973 J. Plasma Phys. (To be published.)

KUEH, H. H., Stewhart, G. E. \& YEH, C. 1965 Phys. Iluids, 8, 723.

LaNdad, L. $1946 J$. Phys. USSR, 10, 25.

Le Coquin, E., Henry, D., Le Meur, J. P., Castrec, C. \& Treguter, J. P. 1971 Rev. Phys. Appliquée, 6, 467.

O'Nmil, T. M. \& Malmbirg, J. H. 1968 Phys. Fluids, 11, 1754.

Treguter, J. P. \& Henry, D. 1972 Plasma Phys. 14, 667. 